\section{Funcionalidad familiar en la adherencia terapéutica del paciente con hipertensión arterial sistémica en primer nivel de atención}

\author{
Family functionality in the therapeutics adherence of the patient \\ with systemic arterial hypertension in the first level of attention
}

Eli Nahim Becerra Partida, ${ }^{* \neq}$ Carolina Villegas Galindo*

\section{RESUMEN}

Introducción: La hipertensión arterial sistémica (HAS) y su prevalencia en México son uno de los principales factores de eventos cardiovasculares, afectando directamente en la calidad de vida. Controlar el padecimiento en primer nivel constituye una estrategia terapéutica que debe impulsar al paciente al buen apego a tratamiento. Es de igual importancia el entorno social del paciente, principalmente el familiar, aportando soporte y seguridad o bien, conflictos y problemáticas que repercuten en la evolución de la enfermedad. Objetivo: Conocer la funcionalidad familiar en la adherencia terapéutica del paciente con hipertensión arterial sistémica en la unidad de medicina familiar Núm. 78, en Guadalajara, Jalisco, México. Material y Métodos: Se realizó un estudio observacional, descriptivo, transversal, prospectivo correlacional en el cual se aplicaron los instrumentos con variables sociodemográficas, así como la Escala de Adherencia Terapéutica para Pacientes Crónicos Basada en Comportamientos Explícitos, y la prueba de percepción del funcionamiento familiar (FF-SIL), a todo paciente hipertenso que acuda a consulta externa de la Unidad de Medicina Familiar Núm. 78 con previo consentimiento informado. El universo contemplado fue de 2,705 pacientes, de los cuales se obtuvo una muestra de 336 pacientes, con un intervalo de confianza de 95\% para un error tipo I del 5\%, expresado con un valor de 0.05. El análisis estadístico se realizó con el software SPSS versión 19, utilizando una estadística descriptiva en la presentación de datos, que incluye las medidas de tendencia central, dispersión más común y porcentajes. Se utilizó prueba H de Kruskal Wallis y Chi cuadrada para las variables intervinientes no paramétricas. Cuando la distribución de ambas variables resulta normal, se utilizó la correlación de Pearson, y en caso contrario se aplicó la correlación de Spearman. Resultados: Se estudió una población total de 336 pacientes; con un rango de edad mayoritario de 60-69 años. En la determinación de la funcionalidad familiar, se aprecia una tendencia en el aumento de la presión arterial sistémica relacionado a la disfunción familiar, siendo una tensión arterial promedio 130/78 mmHg en el grupo que calificó para familia severamente disfuncional. Respecto al tiempo de evolución se observó una tendencia al aumento en años de diagnóstico en relación a la disfuncionalidad familiar, teniendo un promedio de 10.2 años de evolución con HAS, aquellos con familia funcional, en contraste a 12.67 años en familia severamente disfuncional. En las variables de importancia central para el estudio, la adherencia al tratamiento obtuvo una media de 76.4 puntos \pm 8.6. El $89.6 \%$ mostró una alta adherencia al tratamiento y el $10.42 \%$ una adherencia moderada. La escala FF-SIL mostró una frecuencia de $54 \%$ con familia funcional, 37.2\% moderadamente funcional, $7 \%$ disfuncional y 0.9\% severamente disfuncional. Conclusión: El estudio mostró que la buena funcionalidad familiar fomenta y preserva la salud del individuo, lo cual está ligado a la adherencia y buen apego a cambios del estilo de vida, teniendo un impacto significativo para el bienestar grupal e individual por parte del paciente. Ante la relación estrecha observada entre la adherencia terapéutica con la familia, es fundamental presentar un proyecto enfocado a dirigir esfuerzos en materia de prevención.

Palabras clave: Funcionalidad familiar, FF-SIL, adherencia terapéutica, hipertensión arterial sistémica.
* Médico cirujano y partero. Especialidad de Medicina Familiar. Universidad de Guadalajara.

‡Maestría en Ética. Instituto de Ética y Terapia de Jalisco. Doctorando en Bioética y Derechos Humanos. Instituto de Ética y Terapia de Jalisco.

Correspondencia: ENBP, elinahim@hotmail.es Conflicto de intereses: Se manifiesta que no existe ningún potencial conflicto de intereses relacionado con el artículo.

Citar como: Becerra PEN, Villegas CC. Funcionalidad familiar en la adherencia terapéutica del paciente con hipertensión arterial sistémica en primer nivel de atención. Rev CONAMED. 2021; 26(2): 57-67. https://dx.doi. org/10.35366/100348 Financiamiento: Realizado en su totalidad por los autores. Sin financiamiento externo.

Recibido: 06/01/2020. Aceptado: 31/03/2020. 


\begin{abstract}
Introduction: Systemic arterial hypertension and its prevalence in Mexico are one of the main factors of cardiovascular events, directly affecting quality of life. Controlling the disease at the first level of attention constitutes a therapeutic strategy that should encourage the patient to adhere to good treatment. The social environment of the patient is of equal importance, mainly in the familiar environment, providing support and security or, conflicts and problems that affect the evolution of the disease. Objective: To know the family functionality in the therapeutic adherence of the patient with systemic arterial hypertension in the family medicine unit \#78 in Guadalajara, Jalisco, Mexico. Material and Methods: An observational, descriptive, cross-sectional, prospective correlational study was conducted in which sociodemographic variable instruments were applied, as well as the Therapeutic Adherence Scale for Chronic Patients based on Explicit Behaviors, and the test of family functionality perception (FF-SIL), to all hypertensive patients who go to the outpatient clinic of the Family Medicine Unit \#78, with prior informed consent. The universe contemplated was 2705 patients, of which a sample of 336 patients was obtained, with a 95\% confidence interval for a type I error of 5\%, expressed with a value of 0.05. The statistical analysis was performed with the SPSS software version 19, presenting the data in a descriptive statistic, which includes percentages for the most common central tendency and dispersion measures. Kruskal Wallis $\mathrm{H}$ test and Chi square test were used for the non-parametric intervening variables. When the distribution of both variables was normal, Pearson 's correlation was used, and otherwise Spearman's correlation was applied. Results: A total population of 336 patients was studied; with a majority age range of 60-69 years. In determination family functionality, there it a trend in the increase on systemic blood pressure related to family dysfunction, with an average blood pressure being $130 / 78 \mathrm{mmHg}$ in the group that qualify for severely dysfunctional family. Regarding the evolution time, a tendency to increase in years of diagnosis in relation to family dysfunction was observed with an average of 10.2 years of evolution with SAH for those with functional families, in contrast to 12.67 years in a severely dysfunctional family. In the main importance variables for the study, adherence to treatment showed a mean of 76.4 points \pm 8.6. $89.6 \%$ showed high adherence to treatment and $10.42 \%$ showed moderate adherence. The FF-SIL scale showed a frequency of $54 \%$ with functional families, 37.2\% moderately functional, 7\% dysfunctional and $0.9 \%$ severely dysfunctional. Conclusions: The study showed that good family functionality promotes and preserves the health of the individual, which is linked to adherence and good attachment to lifestyle changes, having a significant impact on the group and individual well-being of the patient. Given the close relationship observed between therapeutic adherence with the family, it is essential to present a project focused on directing efforts in the field of prevention to health managers.
\end{abstract}

Keywords: Family functionality, FF-SIL, therapeutic adherence, systemic arterial hypertension.

\section{INTRODUCCIÓN}

La hipertensión arterial sistémica (HAS) es un síndrome de etiología múltiple,' la cual es debida a diversos mecanismos metabólicos. El daño subsecuente y las alteraciones sistémicas son consecuencia del aumento a partir de 115/75 mmHg, duplicando el riesgo cardiovascular por cada 20 $\mathrm{mmHg}$ de presión sistólica y $10 \mathrm{mmHg}$ de la diastólica en individuos de 40-70 años. 2,3

De acuerdo con la Encuesta Nacional de Salud y Nutrición, actualmente, en México, uno de cada cuatro adultos padece HAS. Se ha registrado la estrecha relación de la HAS con la edad, el medio ambiente, el estilo de vida, el géneroy la predisposición genética, lo que provoca un crecimiento en las enfermedades crónico-degenerativas, entre otras, y a su vez contribuyen de manera considerable a la carga de los gastos en el sector sanitario. ${ }^{4}$ En su mayoría serán incapacitantes con un alto grado de mortalidad por complicaciones, como los eventos por EVC, cardiopatía isquémica y alteraciones endocrinometabólicas, neurogénicas y nefropatías. El tratamiento puede modificar el curso de la enfermedad, por lo que deben realizarse controles de las cifras de tensión arterial, la presencia de factores de riesgo y enfermedades concomitantes, así como la presencia de lesión a órganos y la situación personal y social. Por ello, la estrategia terapéutica utilizada debe reforzar con acciones la adecuada adherencia terapéutica a los estilos de vida saludable y al tratamiento farmacológico. ${ }^{5}$ 
Las evidencias científicas demuestran las dificultades que presentan los pacientes hipertensos con la adherencia a estos tratamientos. ${ }^{6}$

La adherencia terapéutica es parte del comportamiento implicado en la salud y la responsabilidad de los individuos con el cuidado y mantenimiento de la misma; por lo que se hace hincapié en diferenciar entre adherencia y cumplimiento al tratamiento, pues la primera requiere la aprobación y participación del paciente en el proceso de elaboración de las recomendaciones, proyectando al paciente como socio activo de los médicos en el proceso de atención. ${ }^{8}$

Diversos son los factores que contribuyen al cumplimiento del tratamiento médico, algunos de ellos tienen que ver con la organización de los servicios de salud, la percepción psicológica, las características del tratamiento, la disponibilidad de recursos económicos, la automedicación y el apoyo familiar. ${ }^{9}$ Mostrándose como dificultad para iniciarlo de forma voluntaria, la suspensión o abandono, la inasistencia a consulta, la ausencia de modificación de hábitos y los estilos de vida. ${ }^{10}$

Para mejorar el cumplimiento terapéutico es importante que el médico explique al paciente su enfermedad, tratamiento, beneficios y efectos adversos, siendo necesario incluir a la familia como eje principal de la interacción individuo-sociedad y fuente principal de apoyo afectivo y emocional para afrontar con éxito los problemas.1,12

La familia satisface las necesidades que están en función de la conservación, fomento y recuperación de la salud; cuando uno de sus integrantes es afectado por alguna enfermedad, la funcionalidad del núcleo familiar se ve amenazada. 5,13

La adecuada sinergia emocional y empatía familiar conllevan al aporte recíproco entre individuo-núcleo como eje de sustentación; caso contrario cuando los integrantes del núcleo mantienen relaciones disfuncionales, las cuales generan desajustes del paciente y el entorno familiar, lo que dificulta el camino a la estabilidad de la enfermedad y pone en juego la adherencia terapéutica, modificando la calidad de vida y de sus familiares. ${ }^{5}$

Una familia funcional, es aquella capaz de cumplir con las necesidades básicas, de acuerdo a la etapa en que se encuentre y en relación con las demandas que percibe del medio ambiente; por lo tanto, una familia funcional es un factor determinante en la conservación de la salud del paciente. ${ }^{5}$

A través del estudio de la funcionalidad dentro del núcleo familiar se obtiene información de suma importancia para valorar la adherencia al tratamiento en una patología específica. Con los resultados obtenidos se puede iniciar una estrategia para brindar el apoyo necesario a estas familias, realizando estrategias de intervención dirigidas a las áreas identificadas como debilitantes o desestabilizadoras, que permitan al clínico en su práctica ofrecerle herramientas a la familia para enfrentar sus problemas.

\section{MATERIAL Y MÉTODOS}

Participantes: el universo estuvo conformado por 2,705 pacientes con hipertensión arterial sistémica que acuden a consulta de medicina familiar en la Unidad de Medicina Familiar Núm. 78 del Instituto Mexicano del Seguro Social, en Guadalajara, Jalisco, México. La muestra calculada fue de 336 pacientes con un intervalo de confianza de $95 \%$ y un margen de error del $5 \%$. Los criterios de inclusión fueron derechohabientes, pacientes con diagnóstico de hipertensión arterial sistémica, que aceptaron voluntariamente y firmaron consentimiento informado. Los criterios de no inclusión fueron pacientes analfabetas, pacientes con enfermedad renal, pacientes con diagnóstico de enfermedad psiquiátrica, pacientes dependientes de familiar para ingesta de medicamento y pacientes que se nieguen a participar en el estudio. Los criterios de eliminación: Ilenado incorrecto del test y consentimiento informado no firmado.

Instrumento: se aplicó el test de funcionalidad familiar FF-SIL que consta de 14 ítems mostrando en cada uno como posibles respuestas: casi nunca, pocas veces, a veces, muchas veces, casi siempre; calificado cada uno con el valor en puntos de: 1, 2, 3, 4, 5 en el orden respectivamente mencionado, obteniendo una sumatoria de 14 puntos como mínima y máxima de 70 puntos para clasificar a la funcionalidad familiar como: funcional (57-70 puntos), moderadamente funcional (43-56 puntos), disfuncional (28-42) y severamente disfuncional (14-27 puntos). Se aplicó también la escala de 
adherencia terapéutica para pacientes crónicos en comportamientos explícitos que consta de 21 ítems, mostrando cada uno como posibles respuestas un valor numérico que va de 0-100\%, obteniendo un máximo de 2,100 puntos y un mínimo de 0 puntos, de lo cual se hace un cociente entre el número total de ítems y se clasifica dependiendo el resultado: baja adherencia al tratamiento (0-33 puntos), moderada adherencia al tratamiento (3467 puntos) y alta adherencia al tratamiento (68-100 puntos). Posterior a esto, se tomó la presión arterial con esfigmomanómetro de mercurio, de acuerdo a las recomendaciones de la American Heart Association (AHA).

Procedimiento: los pacientes contestaron los test voluntariamente, previa firma de consentimiento informado. Una vez concluidas las encuestas se realizó una base de datos. Posteriormente los resultados se analizaron con el software estadístico SPSS, por medio de un análisis descriptivo. El resultado de las variables cualitativas se representó en porcentaje y frecuencia, mientras que las variables cuantitativas se expresan en medidas de

\begin{tabular}{lcrr}
\multicolumn{4}{c}{ Tabla 1: Características sociodemográficas. } \\
\hline Característica & Parámetro & $\mathrm{n}$ & \multicolumn{1}{c}{$\%$} \\
\hline Género & Femenino & 214 & 63.7 \\
& Masculino & 122 & 36.3 \\
Edad (años) & $20-29$ & 3 & 0.9 \\
& $30-39$ & 5 & 1.5 \\
& $40-49$ & 34 & 10.1 \\
& $50-59$ & 81 & 24.1 \\
& $60-69$ & 126 & 37.5 \\
& $70-75$ & 87 & 25.9 \\
& Soltero & 23 & 6.8 \\
& Casado & 237 & 70.5 \\
& Divorciado & 22 & 6.5 \\
& Viudo & 37 & 11.0 \\
& Unión Libre & 17 & 5.1 \\
& Primaria & 118 & 35.1 \\
& Secundaria & 94 & 28.0 \\
& Bachillerato & 69 & 20.5 \\
& Licenciatura & 55 & 16.4 \\
\hline & & &
\end{tabular}

\begin{tabular}{llrr}
\multicolumn{4}{c}{ Tabla 2: Factores de riesgo modificables } \\
\hline Factor & Parámetro & $\mathrm{n}$ & $\%$ \\
\hline \multirow{2}{*}{ Índice de masa corporal } & Normal & 51 & 15.2 \\
& Sobrepeso & 156 & 46.4 \\
& Obesidad & 129 & 38.4 \\
Tabaquismo & Sí & 93 & 27.7 \\
& No & 243 & 72.3 \\
\hline
\end{tabular}

\begin{tabular}{lcc}
\multicolumn{3}{c}{ Tabla 3: Funcionalidad familiar FF-SIL. } \\
\hline Característica & Frecuencia & $\%$ \\
\hline Funcional (70-57) & 184 & 54.8 \\
Moderadamente funcional & 125 & 37.2 \\
$\begin{array}{l}\text { (56-43) } \\
\text { Disfuncional (42-28) }\end{array}$ & 24 & 7.1 \\
Severamente disfuncional & 3 & 0.9 \\
(27-14) & & \\
\hline
\end{tabular}

tendencia central y dispersión. Se aceptó un nivel de confiabilidad de 0.5 en adelante con significancia menor a 0.05 .

\section{RESULTADOS}

Se estudió una población total de 336 pacientes que cumplieron con los criterios de inclusión en la Unidad de Medicina Familiar Núm. 78. Se aplicó un cuestionario en el que se recabaron los datos sociodemográficos de los pacientes (Tabla 7).

De la muestra estudiada 36.3\% pertenece al género masculino y $63.7 \%$ al género femenino. En cuanto a la edad, la media fue de 61.45 años, con una edad mínima de 24 y una máxima de 75 años.

Respecto al estado civil, 70.5\% ( $n=237)$ estaban casados, y en menor porcentaje de frecuencia posterior eran viudos, solteros, divorciados y en unión libre.

La escolaridad muestra un porcentaje decreciente de menor a mayor nivel educativo, se obtuvo $35.12 \%$ primaria y $27.98 \%$ secundaria, el resto con menor número de sujetos corresponde a preparatoria y licenciatura. 
Tabla 4: Funcionalidad familiar FF-SIL y variables intervinientes.

\begin{tabular}{|c|c|c|c|c|c|c|c|c|}
\hline FF-SIL & & Edad & TAS & TAD & Tiempo & Peso & Talla & $\begin{array}{c}\text { Índice de } \\
\text { masa corporal }\end{array}$ \\
\hline \multirow[t]{4}{*}{ Funcional (70-57) } & $\begin{array}{l}\text { Media } \\
\text { Mediana }\end{array}$ & $\begin{array}{r}61.600 \\
64.000\end{array}$ & 124.970 & $\begin{array}{l}74.110 \\
74.000\end{array}$ & $\begin{array}{l}10.200 \\
10.000\end{array}$ & $\begin{array}{l}74.980 \\
74.500\end{array}$ & $\begin{array}{l}1.62490 \\
1.63000\end{array}$ & $\begin{array}{l}28.39050 \\
28.01900\end{array}$ \\
\hline & \multirow{2}{*}{$\begin{array}{l}\text { Varianza } \\
\text { Desviación }\end{array}$} & \multirow{2}{*}{$\begin{array}{r}99.935 \\
9.997\end{array}$} & 151.387 & 133.354 & \multirow{2}{*}{$\begin{array}{c}35.058 \\
5.921\end{array}$} & 163.551 & \multirow{2}{*}{$\begin{array}{l}0.00700 \\
0.08189\end{array}$} & 19.18100 \\
\hline & & & 12.304 & 11.548 & & 12.789 & & 4.37961 \\
\hline & $\mathrm{N}$ & 184.000 & 184.000 & 184.000 & 184.000 & 184.000 & 184.00000 & 184.00000 \\
\hline \multirow{4}{*}{$\begin{array}{l}\text { Moderadamente } \\
\text { funcional (56-43) }\end{array}$} & \multirow{4}{*}{$\begin{array}{l}\text { Media } \\
\text { Mediana } \\
\text { Varianza } \\
\text { Desviación } \\
\text { N }\end{array}$} & $\begin{array}{l}61.040 \\
62.000\end{array}$ & $\begin{array}{l}129.230 \\
130000\end{array}$ & $\begin{array}{l}77.320 \\
78.000\end{array}$ & $\begin{array}{r}11.420 \\
10.000\end{array}$ & $\begin{array}{l}79.660 \\
79.000\end{array}$ & $\begin{array}{l}1.61400 \\
1.61000\end{array}$ & $\begin{array}{l}30.59700 \\
29.74420\end{array}$ \\
\hline & & \multirow{2}{*}{$\begin{array}{r}104.765 \\
10.235\end{array}$} & 198.293 & 71.639 & 40.503 & 190.663 & 0.00800 & 24.36700 \\
\hline & & & 14.082 & 8.464 & 6.364 & 13.808 & 0.08665 & 4.93626 \\
\hline & & 125.000 & 125.000 & 125.000 & 125.000 & 125.000 & 125.00000 & 125.0000 \\
\hline \multirow{5}{*}{$\begin{array}{l}\text { Disfuncional } \\
(42-28)\end{array}$} & \multirow{3}{*}{$\begin{array}{l}\text { Media } \\
\text { Mediana } \\
\text { Varianza }\end{array}$} & \multirow{2}{*}{$\begin{array}{l}60.750 \\
64.000\end{array}$} & 130.460 & 75.330 & 11.380 & 87.630 & 1.60880 & 33.91840 \\
\hline & & & 130.000 & 80.000 & 10.000 & 87.000 & 1.58500 & 33.19880 \\
\hline & & 120.370 & 194.433 & 279.971 & 56.766 & 360.158 & 0.00700 & 53.16700 \\
\hline & Desviación & 13.971 & 13.944 & 16.732 & 7.534 & 18.978 & 0.08088 & 72916.00000 \\
\hline & $\mathrm{N}$ & 24.000 & 24.000 & 24.000 & 24.000 & 24.000 & 24.00000 & 24.00000 \\
\hline Severamente & Media & 74.330 & 130.000 & 78.000 & 12.670 & 83.000 & 1.61000 & 32.02350 \\
\hline disfuncional (27-14) & Mediana & 74.000 & 130.000 & 76.000 & 10.000 & 80.000 & 1.650000 & 32.89330 \\
\hline & Varianza & 0.333 & 100.000 & 124.000 & 41.333 & 63.000 & 0.00500 & 5.42400 \\
\hline & Desviación & 0.577 & 10.000 & 11.136 & 6.429 & 7.937 & 0.06928 & 2.32904 \\
\hline & $\mathrm{N}$ & 3.000 & 3.000 & 3.000 & 3.000 & 3.000 & 3.00000 & 3.00000 \\
\hline Total & Media & 61.450 & 126.990 & 75.430 & 10.760 & 77.690 & 1.61960 & 29.63870 \\
\hline & Mediana & 63.000 & 128.000 & 78.000 & 10.000 & 76.000 & 1.62000 & 28.61310 \\
\hline & Varianza & 103.233 & 175.107 & 121.678 & 38.679 & 197.825 & 0.00700 & 24.74100 \\
\hline & Desviación & 10.160 & 13.233 & 11.031 & 6.219 & 14.065 & 0.08341 & 5.07360 \\
\hline & & 336.000 & 336.000 & 336.000 & 336.000 & 336.000 & 336.00000 & 336.00000 \\
\hline
\end{tabular}

TAS = tensión arterial sistólica; TAD = tensión arterial diastólica.

En cuanto a los factores de riesgo modificables como el índice de masa corporal (IMC), se observó que $46.4 \%$ tiene sobrepeso y $38.4 \%$ obesidad. El $27.7 \%$ de los individuos mostraron resultados positivos para tabaquismo (Tabla 2).

Se observó que, en la población total de estudio, 34.52\% padece DM2.

Respecto al registro de tensión arterial se obtuvieron cifras de control en 81.55\%, con una tensión arterial sistólica (TAS) promedio de $126.9 \mathrm{mmHg}$, mínima de 100 mmHg y máxima de 192 mmHg, y tensión arterial diastólica (TAD) promedio de 76.11
mmHg, con mínima de 58 mmHg y máximo de $108 \mathrm{mmHg}$, con 81.5\% con cifras de control en la toma de tensión arterial. El tiempo de evolución de HAS expresada en años tuvo un promedio de 10.76 años, con un periodo mínimo de 1 y máximo de 35 años de evolución.

\section{Funcionalidad familiar y apego al tratamiento}

La adherencia al tratamiento fue medida de 1 a 100 puntos, directamente proporcional al nivel de 
apego. Se obtuvo una media de 76.4 puntos, con un mínimo de 38 y un máximo de 98 puntos. El 89.6\% corresponde a una alta adherencia al tratamiento y $10.42 \%(n=35)$ a moderada adherencia.

La funcionalidad familiar con base en el puntaje obtenido mediante la escala FF-SIL se califica obteniendo: de 14-27 puntos, severamente disfuncional; de 28-42 puntos, disfuncional; de 43-56 puntos, moderadamente funcional y de 57-70 puntos, funcional (Tabla 3).

Se aprecia una tendencia en el aumento de la presión arterial sistémica relacionado a la disfunción familiar, siendo una tensión arterial promedio de 124/74 mmHg en los individuos que obtuvieron puntaje para familia funcional, $129 / 77 \mathrm{mmHg}$ para el grupo de familia moderadamente funcional, 130/75 mmHg aquéllos con familia disfuncional y 130/78 mmHg en el grupo que calificó para familia severamente disfuncional.

Adicionalmente el tiempo de evolución e IMC denotan una tendencia al aumento en años de diagnóstico e IMC en relación a la disfuncionalidad familiar, teniendo un promedio de 10.2 años de evolución con HAS aquéllos con familia funcional, 11.42 y 11.38 años para familia moderadamente funcional y disfuncional, respectivamente, y, por último, 12.67 en familia severamente disfuncional. Acerca del IMC se obtuvo para la familia funcional un promedio de 28.39, para aquéllas con moderadamente funcional 30.59, disfuncional con 33.91 y las severamente disfuncionales con un promedio de 32.02 (Tabla 4).

El grado de adherencia al tratamiento de los sujetos de estudio muestra una tendencia en el aumento de la presión arterial sistémica relacionado a una menor adherencia terapéutica, registrando una tensión arterial promedio de 128/78 mmHg en la adherencia moderada y $126 / 75 \mathrm{mmHg}$ para quienes obtuvieron una alta adherencia.

Se observó que en el tiempo de evolución la adherencia aumentó respecto a mayor tiempo de ser diagnosticada la patología. Con un promedio de 8.46 años para adherencia moderada y 11.03 años de evolución para sujetos con alta adherencia (Tabla 5).

Debido a que la mayoría de la población de estudio obtuvo un puntaje de alta adherencia, se

\section{Tabla 5: Adherencia terapéutica y variables intervinientes.}

\begin{tabular}{|c|c|c|c|c|c|c|c|c|}
\hline FF-SIL & & Edad & TAS & TAD & Tiempo & Peso & Talla & $\begin{array}{c}\text { Índice de masa } \\
\text { corporal }\end{array}$ \\
\hline \multirow[t]{5}{*}{ Moderada } & Media & 60.030 & 128.000 & 78.740 & 8.460 & 86.110 & 1.66260 & 31.20980 \\
\hline & Mediana & 61.000 & 130.000 & 80.000 & 8.000 & 82.000 & 1.65000 & 29.35750 \\
\hline & Varianza & 100.087 & 203.294 & 78.138 & 36.432 & 298.575 & 0.00500 & 40.25900 \\
\hline & Desviación & 10.004 & 14.258 & 8.840 & 6.036 & 17.279 & 0.06942 & 6.34503 \\
\hline & $\mathrm{N}$ & 35.000 & 35.000 & 35.000 & 35.000 & 35.000 & 35.00000 & 35.00000 \\
\hline \multirow[t]{5}{*}{ Alta } & Media & 61.610 & 126.890 & 75.040 & 11.030 & 76.710 & 1.61460 & 29.45600 \\
\hline & Mediana & 64.000 & 128.000 & 78.000 & 10.000 & 76.000 & 1.61000 & 28.57800 \\
\hline & Varianza & 103.672 & 172.364 & 125.585 & 38.373 & 177.831 & 0.0070 & 23.86000 \\
\hline & Desviación & 10.182 & 13.129 & 11.206 & 6.195 & 13.335 & 0.08355 & 4.88471 \\
\hline & $\mathrm{N}$ & 301.000 & 301.000 & 301.000 & 301.000 & 301.000 & 301.00000 & 301.00000 \\
\hline \multirow[t]{5}{*}{ Total } & Media & 61.450 & 126.990 & 75.430 & 10.760 & 77.690 & 1.61960 & 29.63870 \\
\hline & Mediana & 63.000 & 128.000 & 78.000 & 10.000 & 76.000 & 1.62000 & 28.61310 \\
\hline & Varianza & 103.233 & 175.107 & 121.678 & 38.679 & 197.825 & 0.00700 & 25.74100 \\
\hline & Desviación & 10.160 & 13.233 & 11.031 & 6.219 & 14.065 & 0.08341 & 5.07360 \\
\hline & $\mathrm{N}$ & 336.000 & 336.000 & 336.000 & 336.000 & 336.000 & 336.00000 & 336.00000 \\
\hline
\end{tabular}

TAS = tensión arterial sistólica; TAD = tensión arterial diastólica. 


\begin{tabular}{lccccc}
\multicolumn{7}{c}{ Tabla 6: Adherencia terapéutica y tensión arterial. } \\
\hline Tensión arterial & Media & Mediana & Varianza & Desviación & $n$ \\
\hline$<$ 120/80 & 77.84 & 78.0 & 69.099 & 8.313 & 90 \\
120/80-129/84 & 77.20 & 78.5 & 80.823 & 8.996 & 88 \\
130/85-139/89 & 75.83 & 76.0 & 70.163 & 8.376 & 95 \\
140/90-159/99 & 74.24 & 73.0 & 77.309 & 8.793 & 58 \\
160/100-179/109 & 75.25 & 74.0 & 52.917 & 7.274 & 4 \\
$>$ 180/110 & 87.00 & 87.0 & 0 & 0 & 1 \\
Total & 76.48 & 79.0 & 74.662 & 8.641 & 336 \\
\hline
\end{tabular}

procedió a desestatificar a porcentajes el resultado categórico obtenido equivalente a la puntuación reportada con un rango del $0-100 \%$, resultando con un incremento de la tensión arterial a menor apego al tratamiento (Tabla 6).

Se registró un descenso en el porcentaje de adherencia al tratamiento de acuerdo a la disfuncionalidad familiar (Tab/a 7).

Para la FF-SIL sobre funcionalidad familiar se observó que los valores sobre edad, tensión arterial, IMC y porcentaje de adherencia al tratamiento obtuvieron una $\mathrm{p}<0.05$, por lo tanto, con un nivel de confianza del 95\%, podemos aceptar la hipótesis de que existe diferencia estadísticamente significativa en los valores medios de las variables entre los distintos grupos de funcionalidad familiar, principalmente la variable de adherencia al tratamiento, objetivo inicial en este estudio.

En el grado de adherencia al tratamiento se observó que los valores sobre el tiempo de evolución de la enfermedad obtuvieron una $\mathrm{p}<0.05$, por tanto, con un nivel de confianza del 95\%, podemos aceptar la hipótesis de que existe diferencia estadísticamente significativa en los valores medios de la variable entre los distintos grupos de adherencia al tratamiento.

Para la independencia entre variables de adherencia terapéutica y funcionalidad familiar, como el valor de p fue < 0.05, aceptamos la hipótesis de dependencia entre sí mismas.

En cuanto a la independencia entre variables de funcionalidad familiar y TAS en control (cifras < 140/90 $\mathrm{mmHg}$ ), se obtuvo valor de $\mathrm{p}<0.05$, aceptamos la hipótesis de dependencia entre las mismas.
Para la independencia entre variables de funcionalidad familiar a IMC, el valor de la prueba de independencia sale significativo ( $p<0.05)$, así que aceptamos la hipótesis de dependencia entre las variables. Debido a que hay más de un $25 \%$ de las casillas de la tabla cruzada con frecuencia inferior o igual a cinco no podemos considerar valido el test $\chi^{2}$.

Se observó que en la independencia para las variables de funcionalidad familiar y diabetes mellitus tipo 2 (DM2) como el valor p es < 0.05, se acepta la hipótesis de dependencia entre las variables, mostrando un mejor grado de funcionalidad cuando no se tiene DM2.

De acuerdo a los datos registrados en adherencia terapéutica, funcionalidad familiar, cifras de tensión arterial, IMC y peso se encontró una relación lineal estadísticamente significativa, baja e inversamente proporcional entre la funcionalidad familiar (aumento de disfuncionalidad) y adherencia al tratamiento categórica $(r-0.232$, p $\leq$ 0.05), igualmente para el porcentaje de adherencia obtenido ( $r=-0.211, p \leq 0.05$ ); así mismo que los datos anteriores, pero directamente proporcional a la funcionalidad familiar (aumento de disfuncionalidad) fueron TAS ( $r=0.162, p \leq 0.05)$, TAD ( $r=$ $0.136, p \leq 0.05), I M C(r=0.279, p \leq 0.05)$ y peso $(r=$ $0.17, p \leq 0.05)$

En cuanto al porcentaje de adherencia (1-100\%) se encontró una relación lineal estadísticamente significativa, baja e inversamente proporcional con TAD ( $r=-0.140, p \leq 0.05)$ y peso $(r=-0.197, p=0.05)$.

Respecto a la adherencia terapéutica categórica se encontró una relación lineal estadísticamente 
significativa, directamente proporcional a la variable de tiempo (años transcurridos desde el diagnostico de HAS), ( $r=0.151, p \leq 0.05)$, lo mismo sucede en la relación TAS e IMC ( $r=0.140, p \leq 0.05$ ) (Tabla 8).

\section{DISCUSIÓN}

Al concluir el estudio se encontraron similitudes de los resultados obtenidos con la literatura consultada sobre el apego al tratamiento dependiendo del grado de funcionalidad familiar y sus diversas complicaciones al respecto. ${ }^{3}$ De acuerdo a los resultados es de gran utilidad la determinación de la funcionalidad familiar, debido a que es base y pilar en un mejor apego a la farmacología aplicada, así como en mejor evolución del padecimiento y disminución de las complicaciones y factores que se asocian directamente a la hipertensión arterial.

A diferencia de la bibliografía que refiere que la adherencia disminuye a medida que la terapia se alarga y los tratamientos curativos y rehabilitadores producen tasas más altas que los preventivos, en este estudio se determinó que a mayor tiempo de tratamiento en años, los individuos tienden a un mejor apego al mismo e igualmente a mayor edad se obtuvieron cifras de tensión arterial en control al contrario de individuos en rangos de edad menores a 45 años, dando oportunidad a un nuevo aspecto del paciente geriátrico y su adherencia al tratamiento farmacológico. ${ }^{14}$

La mayoría de los sujetos de este estudio tuvieron un elevado puntaje en adherencia terapéutica, que corresponde de forma proporcional al grado de funcionalidad familiar obtenido, lo cual coincide con el estudio realizado en zonas rurales con pacientes hipertensos que tenían una elevada disfuncionalidad dentro de la familia, asociado a bajo apego y cumplimiento al tratamiento médico, lo cual demuestra que parte clave en el buen control de la hipertensión arterial se debe a la red de apoyo familiar sin crear dependencia, de manera conjunta.

Al relacionar los factores de riesgo tales como tabaquismo, obesidad, tiempo de evolución de la enfermedad se encontró que la obesidad presentó significancia estadística al relacionarla con el mal apego al tratamiento, así como la disfuncionalidad familiar. Esto demuestra que existe un impacto positivo en la implementación de programas institucionales con medidas preventivas para conocer la estabilidad familiar que den soporte al paciente, tal es el caso que dicho tema ha sido estudiado mundialmente en los colegios de medicina familiar, teniendo en cuenta al paciente no sólo como individuo crónico a tratar, sino como sujeto vulnerable en la estabilidad del núcleo, su primer entorno social.1,2,15

Se obtuvieron resultados que no estaban contemplados en los objetivos principales, tales como la mejoría del apego al tratamiento dependiendo de los años de evolución, observando un análisis estadísticamente significativo, lo cual llevó a complementarse con una correlación de Spearman entre las variables años de evolución e IMC, observando una correlación positiva y significativa, con ello se pudo inferir sobre el tiempo de cronicidad de conocimientos al paciente sobre la importancia del autocuidado, monitoreo y control de su enfermedad.

\section{CONCLUSIONES}

Dada su prevalencia en México y el mundo, es importante el estudio de medidas tanto preventivas

Tabla 7: Adherencia terapéutica y funcionalidad familiar.

\begin{tabular}{lcccccc}
\hline FF-SIL & Media & Mediana & Varianza & Desviación & & $\mathrm{n}$ \\
\hline Funcional & 77.88 & 78.0 & 61.115 & 7.818 & 184 \\
Moderadamente funcional & 76.46 & 75.0 & 63.605 & 7.975 & 125 & 24 \\
Disfuncional & 67.29 & 69.5 & 117.607 & 10.845 & 3 & 336 \\
Severamente disfuncional & 65.67 & 62.0 & 250.333 & 15.822 & 8.641 & \\
Total & 76.48 & 76.0 & 74.662 & & & 336 \\
\hline
\end{tabular}


Tabla 8: Correlaciones.

\begin{tabular}{|c|c|c|c|c|c|c|c|c|c|}
\hline \multicolumn{2}{|c|}{ Rho de Spearman } & \multirow{2}{*}{$\frac{\text { FF-SIL }}{1.000}$} & \multirow{2}{*}{$\begin{array}{c}\text { Adherencia } \\
-0.232\end{array}$} & \multirow{2}{*}{$\begin{array}{c}\% \\
\text { adherencia } \\
-0.211\end{array}$} & \multirow{2}{*}{$\frac{\text { TAS }}{0.162}$} & \multirow{2}{*}{$\frac{T A D}{0.136}$} & \multirow{2}{*}{$\begin{array}{r}\text { Tiempo } \\
0.080\end{array}$} & \multirow{2}{*}{$\begin{array}{c}\text { Índice de masa } \\
\text { corporal }\end{array}$} & \multirow{2}{*}{$\begin{array}{c}\text { Peso } \\
0.217\end{array}$} \\
\hline FF-SIL & $\begin{array}{l}\text { Coeficiente de } \\
\text { correlación }\end{array}$ & & & & & & & & \\
\hline & Sig. (bilateral) & NA & 0.000 & 0.000 & 0.003 & 0.012 & 0.144 & 0.000 & 0.000 \\
\hline & $\mathrm{N}$ & 336.000 & 336.000 & 336.000 & 336.000 & 336.000 & 336.000 & 336.000 & 336.000 \\
\hline \multirow[t]{3}{*}{ Adherencia } & $\begin{array}{c}\text { Coeficiente de } \\
\text { correlación }\end{array}$ & -0.232 & 1.000 & 0.511 & 0.014 & -0.081 & 0.151 & -0.075 & -0.167 \\
\hline & Sig. (bilateral) & 0.000 & NA & 0.000 & 0.794 & 0.138 & 0.005 & 0.172 & 0.002 \\
\hline & $\mathrm{N}$ & 336.000 & 336.000 & 336.000 & 336.000 & 336.000 & 336.000 & 336.000 & 336.000 \\
\hline \multirow[t]{3}{*}{$\begin{array}{l}\% \\
\text { adherencia }\end{array}$} & $\begin{array}{l}\text { Coeficiente de } \\
\text { correlación }\end{array}$ & -0.211 & 0.511 & 1.000 & -0.105 & -0.140 & 0.010 & -0.074 & -0.197 \\
\hline & Sig. (bilateral) & 0.000 & 0.000 & NA & 0.054 & 0.010 & 0.861 & 0.178 & 0.000 \\
\hline & N & 336.000 & 336.000 & 336.000 & 336.000 & 336.000 & 336.000 & 336.000 & 336.000 \\
\hline \multirow[t]{3}{*}{ TAS } & $\begin{array}{c}\text { Coeficiente de } \\
\text { correlación }\end{array}$ & 0.162 & -0.014 & -0.105 & 1.000 & 0.390 & 0.092 & 0.140 & 0.207 \\
\hline & Sig. (bilateral) & 0.003 & 0.794 & 0.054 & NA & 0.000 & 0.093 & 0.010 & 0.000 \\
\hline & $\mathrm{N}$ & 336.000 & 336.000 & 336.000 & 336.000 & 336.000 & 336.000 & 336.000 & 336.000 \\
\hline \multirow[t]{3}{*}{ TAD } & $\begin{array}{l}\text { Coeficiente de } \\
\text { correlación }\end{array}$ & 0.136 & -0.081 & -0.140 & 0.390 & 1.000 & 0.021 & 0.195 & 0.252 \\
\hline & Sig. (bilateral) & 0.012 & 0.138 & 0.010 & 0.000 & NA & 0.703 & 0.000 & 0.000 \\
\hline & $\mathrm{N}$ & 336.000 & 336.000 & 336.000 & 336.000 & 336.000 & 336.000 & 336.000 & 336.000 \\
\hline \multirow[t]{3}{*}{ Tiempo } & $\begin{array}{l}\text { Coeficiente de } \\
\text { correlación }\end{array}$ & 0.080 & 0.151 & 0.010 & 0.092 & 0.021 & 1.000 & 0.042 & -0.014 \\
\hline & Sig. (bilateral) & 0.144 & 0.005 & 0.861 & 0.093 & 0.703 & NA & 0.448 & 0.795 \\
\hline & $\mathrm{N}$ & 336.000 & 336.000 & 336.000 & 336.000 & 336.000 & 336.000 & 336.000 & 336.000 \\
\hline \multirow[t]{3}{*}{ IMC } & $\begin{array}{l}\text { Coeficiente de } \\
\text { correlación }\end{array}$ & 0.279 & -0.075 & -0.074 & 0.140 & 0.195 & 0.042 & 1.000 & 0.790 \\
\hline & Sig. (bilateral) & 0.000 & 0.172 & 0.178 & 0.010 & 0.000 & 0.448 & NA & 0.000 \\
\hline & $\mathrm{N}$ & 336.000 & 336.000 & 336.000 & 336.000 & 336.000 & 336.000 & 336.000 & 336.000 \\
\hline \multirow[t]{3}{*}{ Peso } & $\begin{array}{l}\text { Coeficiente de } \\
\text { correlación }\end{array}$ & 0.217 & -0.167 & -0.197 & 0.207 & 0.252 & -0.014 & 0.790 & 1.000 \\
\hline & Sig. (bilateral) & 0.000 & 0.002 & 0.000 & 0.000 & 0.000 & 0.795 & 0.000 & NA \\
\hline & $\mathrm{N}$ & 336.000 & 336.000 & 336.000 & 336.000 & 336.000 & 336.000 & 336.000 & 336.000 \\
\hline
\end{tabular}

TAS = tensión arterial sistólica; TAD = tensión arterial diastólica; NA = no aplica.

como de mantenimiento en la población, dando a conocer las distintas redes de apoyo sustentadas en la familia, la cual posee influencia directa en el desarrollo del proceso salud-enfermedad, ya sea al favorecer el apego al tratamiento tanto médicofarmacológico como cambios en el estilo de vida o precipitar complicaciones a largo plazo.
Se obtuvo un resultado panorámico en el que se observaron aspectos relacionados con las características de las organizaciones de salud, la comunicación entre el paciente y el personal sanitario, el tipo de terapéutica establecida, las prescripciones médicas, los conocimientos que tiene la persona acerca de la hipertensión arterial, la escolaridad y el 
tiempo de evolución de la enfermedad para tener un mejor rango de cifras a nivel tensional.

Si bien las variables iniciales de estudios iban encaminadas a reforzar y conocer el poder que tiene la familia en influir al apego y control de la enfermedad, surgieron diversos resultados que no sólo apoyan dicha teoría, sino que abren paso a diversos cuestionamientos, tales como la obesidad en familias disfuncionales o el apego farmacológico a mayor cronicidad de la misma, así como el principal aspecto que se calificaba para tener una buena adherencia, que se refería al abandono de actividades placenteras que eran consideradas como malos hábitos alimenticios, en la cual $80 \%$ de los pacientes respondió que no estaban dispuestos a abandonar el tabaco o que no deseaban realizar actividad física.

Este estudio deja claro que la buena funcionalidad familiar fomenta y preserva la salud del individuo, lo cual está ligado a la adherencia y buen apego a los cambios del estilo de vida, teniendo un impacto significativo para el bienestar grupal e individual por parte del paciente.

Ante la relación estrecha observada que guarda la adherencia terapéutica con la familia, es fundamental presentar a los directivos en salud un proyecto enfocado a dirigir esfuerzos en materia de prevención, que refleje una estrategia que permita abordar la temática como una nueva implementación en mejorar la calidad de vida del paciente y disminución de costos a largo plazo en el sector salud.

\section{AGRADECIMIENTOS}

Al Instituto Mexicano del Seguro Social, como la institución más noble de México.

A nuestras familias, como la célula fundamental de la sociedad y pilar de nuestro crecimiento.

\section{Biblografía}

1. Diagnóstico y Tratamiento de la hipertensión arterial en el Primer Nivel de Atención México: Instituto Mexicano del Seguro Social; 08/07/2014 [Acceso el 29 Ago 2018]. Disponible en: http://www.cenetec-difusion.com/CMCPC/ IMSS-076-08/ER.pdf.

2. Whelton PK, Carey RM, Aronow WS, Casey DE Jr, Collins KJ, Dennison HC et al. 2017 Guideline for the prevention, detection, evaluation, and management of high blood pressure in adults. J Am Coll Cardiol. 2017, 23976; doi: 10.1016/j.jacc.2017.07.

3. James PA, Oparil S, Carter BL, Cushman WC, DennisonHimmelfarb C, Handler J et al. 2014 evidence-based guideline for the management of high blood pressure in adults: report from the panel members appointed to the Eighth Joint National Committee (JNC 8). JAMA. 2014; 311 (5): 507-520. doi: 10.1001/jama.2013.284427.

4. Rosas-Peralta M, Palomo-Piñón S, Borrayo-Sánchez G, Madrid-Miller A, Almeida-Gutiérrez E, H Galván OH et al. Consenso de hipertensión arterial sistémica en México. Rev Med Inst Mex Seguro Soc. 2016; 54 (Supl 1): S7-10.

5. Olvera AS, Müggenburg M, Vigil R. Adherencia terapéutica y funcionalidad familiar en personas con hipertensión arterial. Biblioteca Las Casas, 2014; 10 (2). Disponible en http://www.index-f.com/lascasas/ documentos/lc0771.php.

6. Cazorla MN, Rodríguez D. Grado de adherencia terapéutica a los fármacos del metabolismo óseo-mineral: ¿toman nuestros pacientes la medicación prescrita? Enferm Nefrol [Revista en línea]. 2013 [Acceso agosto 2018]; 16 (1): 7. Disponible en: http://scielo.isciii.es/pdf/enefro/v16nt/07_ original6.pdf.

7. Libertad de Los Ángeles M, Grau JA, Espinosa AD. Marco conceptual para la evaluación y mejora de la adherencia a los tratamientos médicos en enfermedades crónicas. Rev Cubana Salud Pública [En línea]. 2014 [acceso agosto 2018]; 40 (2): 3-4. Disponible en: http://www.revsalud publica.sld.cu/index.php/spu/rt/ printerFriendly/46/32.

8. Jiménez L, Siverio D, Chala JM, Brito Y, Armada Z. Factores relacionados con la no adherencia al tratamiento farmacológico en hipertensos del Policlínico XX Aniversario. Diseño de un programa educativo. CorSalud [Revista en línea]. 2017 [acceso agosto 2018]; 9 (1): 2-3. Disponible en: http://www.revcorsalud.sld.cu/index.php/cors/rt/ printerFriendly/217/588.

9. Pomares AJ, Vázquez MA, Ruíz ES. Adherencia terapéutica en pacientes con hipertensión arterial. Rev Finaly [En línea]. 2017 [Acceso Agosto 2018]; 7 (2): 3-4. Disponible en: http://scielo.sld.cu/scielo.php?script=sci_arttext\&pid =S2221-24342017000200003.

10. Montoya J, Calvache R. Reflexiones en torno a la adherencia al tratamiento. Revista Psicoespacios. 2014; 8 (13): $256-$ 273. Disponible en http://revistas.iue.edu.co/index.php/ Psicoespacios.

11. Conzáles-Castro P. El apoyo familiar en la adherencia al tratamiento nutricional del paciente con diabetes mellitus tipo 2 (DM2). Waxapa [En línea]. 2011 [acceso agosto 2018]; 2 (5): 1-5. Disponible en: www.medigraphic.com/pdfs/ waxapa/wax-2011/wax115e.pdf.

12. Cid MC, Montes de Oca R, Hernandez O. La familia en el estado de salud. Rev Med Electrón [En línea]. 
2014 [Acceso agosto 2018]; 36 (4): 1-7. Disponible en: http://scielo.sld.cu/scielo.php?script=sci_arttext\&pid =S1684-18242014000400008.

13. Barquera S, Campos-Nonato I, Hernández-Barrera L, Villalpando S, Rodríguez-Cilabert C, Durazo-Arvizu R et al. Hypertension in Mexican adults: results from the National Health and Nutrition Survey 2006. Salud Publica de Mexico. 2010; 52 (S1): S63-S71.
14. Arévalo MT, Claro NO, Rotela CA. Relación entre conocimientos en hipertensión arterial y factores de riesgo cardiovasculares con estilos de vida saludables. Revista virtual de postgrado. Facultad de Medicina; Universidad Nacional de Itapúa. 2016; 1 (1): 5-7.

15. Jameson JL, Kasper DL, Longo DL, Fauci AS, Hauser SL, Loscalzo J. Harrison, principios de medicina interna. 20a ed. McGrawHill; 2018. 